\title{
Application of ortho-acylated phenylacetic acid esters to the synthesis of 1-substituted isochromanes
}

\author{
Iliyan Ivanov,* Stoyanka Nikolova, Ekaterina Kochovska, and Stela Statkova-Abeghe \\ University of Plovdiv, Department of Organic Chemistry, 24, Tsar Assent str., 4000 Plovdiv, \\ Bulgaria \\ E-mail:ivanov@argon.acad.bg
}

\begin{abstract}
A series of 1-substituted isochromanes has been synthesized from the corresponding alkyl 2acylphenylacetates $\mathbf{3}$, by reduction and cyclization with a catalytic amount of $p$-toluenesulfonic acid monohydrate (PTSA), as compounds with potential antitumor activity and N-bearing heterocycle analogues.
\end{abstract}

Keywords: Isochromanes, synthesis, alkyl 2-acylphenylacetates, reduction, sodium borohydride

\section{Introduction}

The biological activity of some isochromane derivatives has provided a great deal of interest in the synthesis. The isochromane fragment is the core of various natural compounds, which exhibit a wide variety of pharmacological activities. ${ }^{1}$

Many isochromanes have been synthesized as intermediates of the synthesis of anxiolytic 2,3-benzodiazepines, which are known as 2-amino-3-(3-hydroxy-5-methylisoxazol-4yl)propionic acid (AMPA) receptor antagonists. ${ }^{2-4}$ Recently, Nergardh et al. reported that isochroman A 68930 is a selective dopamine D1 antagonist. ${ }^{5}$ Some isochromanes are pharmacologically active compounds in anti-migraine therapy. For example, PNU-109291 is selective 5-HT1D receptor agonist with anti-migraine potential, like sumatriptan. ${ }^{6}$ Isochromane derivatives are synthesized as aromatic analogues of Stavudine, an approved anti-Human Immunodeficiency Virus (HIV) drug. ${ }^{7}$ The isochromane analogues of the naphthylisoquinoline alkaloids michellamines and korupensamines have been synthesized because of their activity against HIV. ${ }^{8}$

Some isochromane analogues are part of complex natural products such as stephaoxocanine ${ }^{9}$ and glucoside $\mathrm{B} .{ }^{10}$ Isochromane derivatives also exhibit plant-growth regulatory and herbicidal activities; ${ }^{11,12}$ they are estrogen receptors, ${ }^{13}$ dopamine receptor ligands, ${ }^{14}$ and fragrances, such as galaxolide. ${ }^{15}$ 6,7-Dimethoxyisochromanes substituted at C-1 via a one- to three- carbon chain 
with arylpiperazines, $p$-trifluoromethylphenyl, etc., are hypotensives, which lower blood pressure presumably by both peripheral and central $\alpha$-adrenoreceptor blockade. ${ }^{14} 1$-Phenyl- and 1-(3methoxy-4-hydroxy)phenyl-6,7-dihydroxyisochromans have been identified in extra-virgin olive oil and have been shown to exhibit beneficial antioxidant effects ${ }^{16,17}$ and antiplatelet activity. ${ }^{18} 1$ Aryl-6,7-dimethoxyisochromanes have shown a wide range of biological activities such as analgesic, muscle relaxant, antidepressant, anti-inflammatory, antihistaminic and anticoagulant, hypotensive with peripheral and central activities, and are adrenergic antagonists. ${ }^{19-21}$ Heterocyclic anthracyclines, as idarubicin's analogues, include the isochromane nucleus, have been reported to possess a potent and broad spectrum of antitumor activity. ${ }^{22} 1,6,8$-Trihydroxy3-heptyl-7-carboxyisochroman, an isochromane derivative from Penicillium sp. is an antibiotic and a topoisomerase II inhibitor. ${ }^{23}$ Another derivative, pseudoflectusin, is a selective human cancer cytotoxin found in nature from Aspergillus pseudodeflectus. ${ }^{24}$

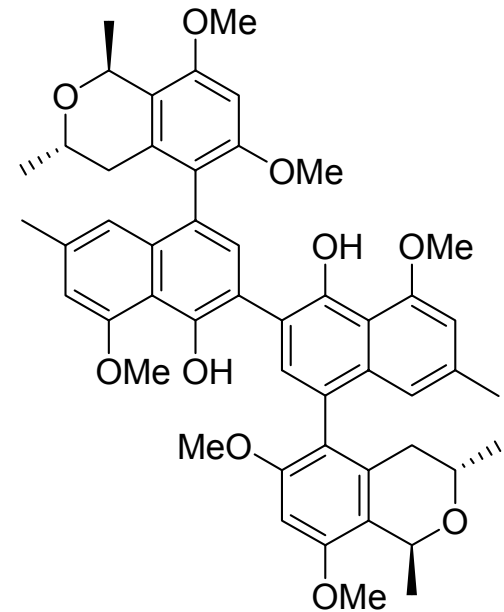

Figure 1. Structure of a synthetic analog of a korupensamine

\section{Results and Discussion}

Isochroman derivatives are structural analogues of tetrahydroisoquinolines and have been repeatedly recognized as such. ${ }^{25,26}$ This analogy has been exploited and several studies report on the use of isochromans as starting materials or intermediates for the synthesis of iso-quinoline derivatives and vice versa, as well as for the preparation of other nitrogen-containing heterocycles. $^{26}$ Numerous methods have been developed to synthesize isochromanes by intramolecular C-O ${ }^{7,27-29}$ or C-C bond ${ }^{30,31}$ cyclization. Recently, the synthesis of isochromanes containing hydroxy substituents has been achieved. The key step for the synthesis of the isochromane nucleus is an oxidative mercury- mediated ring closure of phenylmethanol derivatives or the ozonolysis of the corresponding phenylmethanol derivative. ${ }^{32,33}$ The hydroxyisochromanes were prepared also by saponification of 5-formylmellein, ${ }^{34}$ reduction of 
isocoumarins with hydrides, ${ }^{35,36}$ reaction of benzocyclobutenols and aromatic aldehydes in the presence of lithium 2,2,6,6-tetramethylpiperidide, ${ }^{37}$ photo-induced hetero- Diels-Alder reaction of 2-methylbenzldehydes, ${ }^{38}$ oxidation of 2-(2-hydroxyethyl)-5-isopropylbenzyl alcohol, ${ }^{39}$ 2hydroxymethyl-1-(2'-hydroxyphenyl)naphthalene ${ }^{40}$ with non-activated manganese dioxide or pyridinium chlorochromate, or by reaction of an alcohol and triethyl orthoformate catalyzed by aluminum chloride followed by catalytic hydrogenation. ${ }^{41}$ Other authors also prepared isochroman derivatives by refluxing diols in $50 \%$ aq. $\mathrm{H}_{3} \mathrm{PO}_{4}{ }^{42}$ or $85 \% \mathrm{H}_{3} \mathrm{PO}_{4}{ }^{43}$ in toluene in good- to very good yields.

Isochromanes have been synthesized from acetals of phenethyl alcohols, followed by cyclization utilizing a Lewis acid or via oxa-Pictet-Spengler reaction from 2-phenylethanol and an aldehyde or ketone with a fatty acid as catalyst. ${ }^{26,44,45}$ In addition, some authors used reduction of oximes followed by intramolecular cyclization, ${ }^{46}$ condensation of 3,4dimethoxyphenethyl alcohol with aromatic aldehydes, ${ }^{2-4}$ or cyclization with $p$-toluenesulfonic acid. $^{47}$

In a search for new approaches to the synthesis of isochromanes, we found that they can be obtained from the corresponding ethyl- (or methyl-) 2-acylphenylacetates 3 in very good yields. In our previous reports, we have shown that different ethyl 2-acyl-4,5-dimethoxyphenylacetates can be obtained from ethyl 3,4-dimethoxyphenylacetate and carboxylic acids in the presence of $\mathrm{P}_{2} \mathrm{O}_{5}{ }^{48} \mathrm{We}$ found that the acylation reaction in phenylacetic acid esters takes place if electrondonating groups exist on a third position of the aromatic ring in order to activate it. According to this procedure, we obtained the corresponding alkyl 2-acylphenylacetates from esters of 3methoxy- and 3,4-dimethoxyphenylacetic acid, with very good yields (Scheme 1, Table 1).

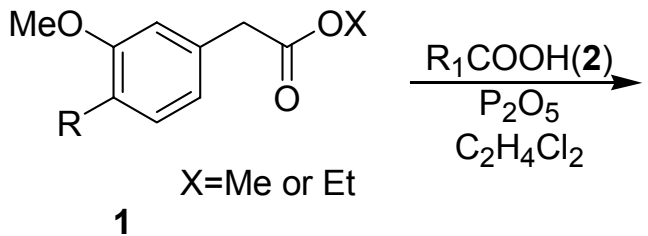<smiles>[R]c1cc(C([R])O)c(CCO)cc1OC</smiles><smiles></smiles>

3<smiles>[R]c1cc2c(cc1OC)CCOC2[R]</smiles>

\section{Scheme 1}


Table 1. Synthesis of alkyl 2-acylphenyl acetates

\begin{tabular}{|c|c|c|c|c|c|c|c|c|c|}
\hline No & $\mathrm{R}$ & $\mathrm{R} 1$ & $\begin{array}{l}\text { Yield* } \\
\%\end{array}$ & $\begin{array}{l}\mathrm{mp} \\
{ }^{\circ} \mathrm{C}\end{array}$ & No. & $\mathrm{R}$ & $\mathrm{R} 1$ & $\begin{array}{c}\text { Yield } \\
* \\
\% \\
\end{array}$ & $\begin{array}{l}\mathrm{mp} \\
{ }^{\circ} \mathrm{C}\end{array}$ \\
\hline $3 a$ & $\mathrm{OMe}$ & $\mathrm{Me}$ & 84 & $94 \mathrm{a}$ & $3 \mathrm{i}$ & $\mathrm{OMe}$ & 2,4- $\mathrm{Cl} 2 \mathrm{C} 6 \mathrm{H} 3$ & 82 & $98-99.5$ \\
\hline $3 b$ & $\mathrm{H}$ & $\mathrm{Me}$ & 77 & $84-87$ & $3 \mathrm{j}$ & $\mathrm{OMe}$ & $\mathrm{Bn}$ & 62 & $73-75 a$ \\
\hline $3 \mathrm{c}$ & $\mathrm{OMe}$ & Et & 79 & $90-94$ & $3 \mathrm{k}$ & $\mathrm{OMe}$ & p-MeOC6H4CH2 & 75 & $83-86$ \\
\hline $3 d$ & $\mathrm{OMe}$ & $\mathrm{i}-\mathrm{Bu}$ & 86 & $\begin{array}{l}71- \\
74 a\end{array}$ & 31 & $\mathrm{OMe}$ & $\begin{array}{c}3,4- \\
(\mathrm{MeO}) 2 \mathrm{C} 6 \mathrm{H} 3 \mathrm{CH} 2\end{array}$ & 80 & $127-129 b$ \\
\hline $3 e$ & $\mathrm{OMe}$ & $\mathrm{n}-\mathrm{C} 15 \mathrm{H} 31$ & 90 & $82-83$ & $3 \mathrm{~m}$ & $\mathrm{OMe}$ & naphth-2-yl & 80 & $54-55$ \\
\hline $3 \mathrm{f}$ & $\mathrm{OMe}$ & $\mathrm{Ph}$ & 90 & $\begin{array}{l}78- \\
79 \mathrm{a}\end{array}$ & $3 n$ & $\mathrm{OMe}$ & $\mathrm{CHPh} 2$ & 60 & $111.5-116$ \\
\hline $3 g$ & $\mathrm{OMe}$ & 3,4-(MeO)2C6H3 & 83 & $\begin{array}{l}113- \\
114\end{array}$ & 30 & $\mathrm{H}$ & $\mathrm{CHPh} 2$ & 65 & $117.5-119$ \\
\hline $3 \mathrm{~h}$ & $\mathrm{OMe}$ & 3,4,5-(MeO)3C6H2 & 74 & $\begin{array}{l}128- \\
130 \\
\end{array}$ & & & & & \\
\hline
\end{tabular}

* The yields were for products isolated after recrystallization.

${ }^{\mathrm{a}} \mathrm{mp}$ and spectroscopic data are given in ref. $48 .{ }^{\mathrm{b}} \mathrm{mp} 125-129^{\circ} \mathrm{C}$ in ref. 46.

The next step in this synthesis of isochromanes was the reduction of the alkyl 2acylphenylacetates, 3. We reduced the alkyl 2-acylphenylacetates to the corresponding diols using sodium borohydride-methanol system. Sodium borohydride under normal conditions does not reduce ester groups but reduces (in moderate- to good yields) double bonds in compounds, where the double bond is cross-conjugated with two carboxylic groups or where the double bond is conjugated with one carboxylic and one phenyl group. ${ }^{49}$ In these cases the reduction was carried out by using equimolar quantities of sodium borohydride and the ester in ethanol or isopropyl alcohol to react first at $0-5^{\circ} \mathrm{C}$ and then at RT.

We found that the reduction of the alkyl 2-acylphenylacetates 3 with sodium borohydride in methanol easily led to the corresponding 2-hydroxy-4,5-dimethoxyphenyl-ethanols 4 with 85$90 \%$ yields (Table 2). Their cyclization in the presence of a catalytic amount of PTSA for $30 \mathrm{~min}$ at RT in dichloromethane afforded the corresponding isochromanes $\mathbf{5}$ (Table 2). 
Table 2. Reduction of alkyl 2-acylphenylacetates to 2-hydroxy-4,5-dimethoxyphenyl-ethanols followed by cyclization with PTSA to isochromanes

\begin{tabular}{|c|c|c|c|c|c|c|c|}
\hline \multicolumn{5}{|c|}{ 2-Hydroxy-4,5-dimethoxyphenyl ethanols } & \multicolumn{3}{|c|}{ Isochromanes } \\
\hline No. & $\mathbf{R}$ & $\mathbf{R}^{1}$ & $\begin{array}{c}\text { Yield } \\
\%\end{array}$ & $\begin{array}{l}\mathrm{mp} \\
{ }^{\circ} \mathrm{C}\end{array}$ & No. & $\begin{array}{c}\text { Yield } \\
\%\end{array}$ & $\begin{array}{l}\mathbf{m p} \\
{ }^{\circ} \mathrm{C}\end{array}$ \\
\hline $4 a$ & OMe & $\mathrm{Me}$ & 94 & $105-105.5$ & $5 \mathbf{a}$ & 92 & Oil \\
\hline $4 b$ & $\mathrm{H}$ & $\mathrm{Me}$ & 97 & $87-88$ & $\mathbf{5 b}$ & 93 & Oil \\
\hline $4 c$ & $\mathrm{OMe}$ & Et & 95 & Oil & $5 c$ & 90 & Oil \\
\hline $4 d$ & $\mathrm{OMe}$ & $\mathrm{i}-\mathrm{Bu}$ & 92 & $49-50$ & $5 d$ & 90 & $118-120$ \\
\hline $4 e$ & $\mathrm{OMe}$ & $\mathrm{n}-\mathrm{C}_{15} \mathrm{H}_{31}$ & 95 & $72-75$ & $5 e$ & 92 & $73-75$ \\
\hline $4 f$ & $\mathrm{OMe}$ & $\mathrm{Ph}$ & 90 & $110-110.5$ & $5 f$ & 88 & $73-75^{\mathrm{a}}$ \\
\hline $4 \mathrm{~g}$ & OMe 3 & $4-(\mathrm{MeO})_{2} \mathrm{C}_{6} \mathrm{H}_{3}$ & 92 & $100-105$ & $5 \mathrm{~g}$ & 85 & $80-82^{b}$ \\
\hline $4 h$ & OMe & $\begin{array}{c}3,4,5- \\
(\mathrm{MeO}){ }_{3} \mathrm{C}_{6} \mathrm{H}_{2}\end{array}$ & 93 & $86-90$ & $5 h$ & 85 & 99-101 \\
\hline $4 i$ & $\mathrm{OMe}$ & $2,4-\mathrm{Cl}_{2} \mathrm{C}_{6} \mathrm{H}_{3}$ & 95 & $151-153$ & $5 \mathbf{i}$ & 87 & Oil \\
\hline $4 \mathbf{j}$ & $\mathrm{OMe}$ & $\mathrm{Bn}$ & 89 & $107-108$ & $5 \mathbf{j}$ & 90 & Oil \\
\hline $4 k$ & OMe $p$ & $-\mathrm{MeOC}_{6} \mathrm{H}_{4} \mathrm{CH}_{2}$ & 88 & $78-79$ & $\mathbf{5 k}$ & 92 & Oil \\
\hline 41 & $\mathrm{OMe}$ & $\mathrm{MeO})_{2} \mathrm{C}_{6} \mathrm{H}_{3} \mathrm{CH}_{2}$ & 85 & $134-136$ & 51 & 90 & $69-72^{c}$ \\
\hline $4 m$ & $\mathrm{OMe}$ & 2-naphthyl & 85 & $124-125$ & $5 \mathrm{~m}$ & 90 & $128-129$ \\
\hline $4 n$ & $\mathrm{OMe}$ & $\mathrm{CHPh}_{2}$ & 88 & $107-109$ & $5 n$ & 88 & $144-154$ \\
\hline 40 & $\mathrm{H}$ & $\mathrm{CHPh}_{2}$ & 92 & $112.5-114$ & 50 & 90 & $91-91.5$ \\
\hline
\end{tabular}

${ }^{\mathrm{a}} \mathrm{mp} 74-75^{\circ} \mathrm{C}$ in ref. $40 ;{ }^{\mathrm{b}} \mathrm{mp} 80-81^{\circ} \mathrm{C}$ in ref. $16 ;{ }^{\mathrm{c}} \mathrm{mp} 69-71^{\circ} \mathrm{C}$ in refs. 25,46

In conclusion, six-membered oxygen-containing heterocycles with the isochromane skeleton were synthesized, as a type of compound found in nature and among bioactive compounds of interest. We have developed a convenient method for their synthesis by simple reduction of ethyl (or methyl) 2-acylphenylacetates in a sodium borohydride-methanol system, followed by cyclization in a less acidic medium with a catalytic amount of PTSA. A variety of substituents at the 1-position of the isochromane skeleton was introduced readily by changing the carboxylic acids. Some of the so-obtained isochromanes are O-analogues of isoquinoline alkaloids: 5l, the O-analogue of papaverine, $\mathbf{5} \mathbf{g}$ and $\mathbf{5 h}$ of cryptostiline II and III, 5a of salsolidine. 


\section{Experimental Section}

General Procedures. Melting points were determined on a Boetius hot-stage apparatus and are uncorrected. IR spectra were measured (KBr pellets or film) with a Perkin-Elmer $1750 \mathrm{IFTS} .{ }^{1} \mathrm{H}$ NMR and ${ }^{13}$ C-NMR were recorded on a Bruker Avance DRX250 or Bruker Avance II+ 600 instrument, using $\mathrm{CDCl}_{3}$ as solvent. Chemical shifts $(\delta, \mathrm{ppm})$ were referenced to TMS $(\delta=0.00$ ppm) as an internal standard and coupling constants are indicated in Hz. All the NMR spectra were taken at RT $(295 \mathrm{~K})$. MS were recorded on a Jeol JMS-D300 spectrometer $(70 \mathrm{eV})$. Elemental analyses were performed in the analytical laboratory at the Faculty of Chemistry, University of Plovdiv. TLC was carried out on precoated $0.2 \mathrm{~mm}$ Fluka silica gel 60 plates, using chloroform/diethyl ether/n-hexane: $6 / 3 / 1$ as chromatographic system. Merck silica gel $60(0.063-$ $0.2 \mathrm{~mm}$ ) was used for chromatographic filtration.

\section{Preparation of alkyl 2-acyl-phenylacetates (Table 1, 3a-o). General procedure. ${ }^{48}$}

To a solution of ethyl (or methyl) 3,4-dimethoxyphenylacetate $(10 \mathrm{mmol})$ and the corresponding carboxylic acid $2(10 \mathrm{mmol})$ in 1,2-dichloroethane $(30 \mathrm{~mL})$ was added $\mathrm{P}_{2} \mathrm{O}_{5}(5 \mathrm{~g})$. The suspension was stirred vigorously for 8-10 $\mathrm{h}$ at RT, then water was added dropwise to the cooled mixture. The organic layer was washed with water $(50 \mathrm{ml}), \mathrm{Na}_{2} \mathrm{CO}_{3}(30 \mathrm{ml})$, water $(50 \mathrm{ml})$ and dried $\left(\mathrm{Na}_{2} \mathrm{SO}_{4}\right)$, then the combined extracts were dried $\left(\mathrm{Na}_{2} \mathrm{SO}_{4}\right)$ and filtered through a short column with neutral $\mathrm{Al}_{2} \mathrm{O}_{3}$. The products, after the removal of the solvent, were purified by

recrystallization with $\mathrm{MeOH}$. Compounds 3a,d,f,j, , I were previously characterized. ${ }^{46,48}$ The spectroscopic data of new compounds follow.

Methyl 2-(2-acetyl-5-methoxyphenyl)acetate (3b). ${ }^{1} \mathrm{H}-\mathrm{NMR}(600 \mathrm{MHz}): 2.55$ (s, 3H), 3.71 (s, $3 \mathrm{H}), 3.86(\mathrm{~s}, 3 \mathrm{H}), 3.93(\mathrm{~s}, 2 \mathrm{H}), 6.76(\mathrm{~d}, 1 \mathrm{H}, J=2.2), 6.87(\mathrm{dd}, 2 \mathrm{H}, J=8.6,2.3), 7.86(\mathrm{~d}, 1 \mathrm{H}$, $J=8.7) ;{ }^{13} \mathrm{C}-\mathrm{NMR}(150.9 \mathrm{MHz}): 199.1,171.8,162.1,139.6,132.8,118.8,111.9,60.7,55.4,51.9$, 40.9. MS $m / z: 236(\mathrm{M}+)$.

Ethyl 2-(4,5-dimethoxy-2-propionylphenyl)acetate (3c). ${ }^{1} \mathrm{H}-\mathrm{NMR}(600 \mathrm{MHz}): 1.18(\mathrm{t}, 3 \mathrm{H}$, $J=7.2$ ), 1.28 (t, 3H, $J=7.2$ ), 2.95 (q, 2H, $J=7.3$ ), 3.89 (s, 2H), 3.93 (s, 6H), 4.17 (q, 2H, $J=7.1$ ), $6.73(\mathrm{~s}, 1 \mathrm{H}), 7.32(\mathrm{~s}, 1 \mathrm{H}) ;{ }^{13} \mathrm{C}-\mathrm{NMR}(150.9 \mathrm{MHz}): 202.0,171.8,151.5,147.3,129.3,129.1$, $115.3,112.8,60.7,56.2,55.9,40.3,33.6,14.3,8.5$. MS m/z: $280\left(\mathrm{M}^{+}\right)$.

Methyl 2-(4,5-dimethoxy-2-palmitoylphenyl)acetate (3e). ${ }^{1} \mathrm{H}-\mathrm{NMR}(600 \mathrm{MHz}): 0.88(\mathrm{t}, 3 \mathrm{H}$, $J=7.1), 1.21-1.36(\mathrm{~m}, 24 \mathrm{H}), 1.67-1.71(\mathrm{~m}, 2 \mathrm{H}), 2.89$ (t, $2 \mathrm{H}, J=7.5), 3.71(\mathrm{~s}, 3 \mathrm{H}), 3.92(\mathrm{~s}, 2 \mathrm{H})$, $3.94(\mathrm{~s}, 6 \mathrm{H}), 6.73(\mathrm{~s}, 1 \mathrm{H}), 7.32(\mathrm{~s}, 1 \mathrm{H}) ;{ }^{13} \mathrm{C}-\mathrm{NMR}$ (150.9 MHz): 201.8, 172.3, 151.6, 147.4, 129.3, 129.1, 115.4, 112.9, 56.2, 55.9, 51.9, 40.5, 40.2, 31.9, 29.7, 29.6, 29.4, 24.5, 22.7, 14.2. MS $m / z: 462\left(\mathrm{M}^{+}\right)$.

Ethyl 2-(2-(3,4-dimethoxybenzoyl)-4,5-dimethoxyphenyl)acetate (3g). ${ }^{1} \mathrm{H}-\mathrm{NMR}(250 \mathrm{MHz})$ : $3.68(\mathrm{~s}, 3 \mathrm{H}), 3.85$ (s, 3H), $3.87(\mathrm{~s}, 6 \mathrm{H}), 3.94(\mathrm{~s}, 3 \mathrm{H}), 4.12(\mathrm{~s}, 2 \mathrm{H}), 6.94(\mathrm{~s}, 1 \mathrm{H}), 7.06(\mathrm{~s}, 1 \mathrm{H})$, 7.10-7.20 (m, 3H): ${ }^{13} \mathrm{C}-\mathrm{NMR}(62.9 \mathrm{MHz}): 196.3,171.2,152.8,149.5,148.3,142.2,133.4,130.6$, $130.3,128.1,114.6,113.4,107.8,61.3,56.2,56.1,38.3,14.1 . \mathrm{MS} m / z: 388\left(\mathrm{M}^{+}\right)$. 
Ethyl 2-(4,5-dimethoxy-2-(3,4,5-trimethoxybenzoyl)phenyl)acetate (3h). ${ }^{1} \mathrm{H}$-NMR $\quad(600$ MHz): 1.18 (t, 3H, J=7.1), 3.78 (s, 2H), $3.83(\mathrm{~s}, 3 \mathrm{H}), 3.86(\mathrm{~s}, 6 \mathrm{H}), 3.94(\mathrm{~s}, 3 \mathrm{H}), 3.97$ (s, 3H), 4.08 (q, 2H, J=7.1), $6.87(\mathrm{~s}, 1 \mathrm{H}), 6.98(\mathrm{~s}, 1 \mathrm{H}), 7.06(\mathrm{~s}, 2 \mathrm{H}) ;{ }^{13} \mathrm{C}-\mathrm{NMR}(150.9 \mathrm{MHz}): 196.1,171.5$, $152.9,150.9,146.9,142.2,133.4,130.3,128.0,114.5,113.4,107.7,60.9,56.3,56.2,56.1,38.7$, 14.2. MS $m / z$ : $418\left(\mathrm{M}^{+}\right)$.

Ethyl 2-(2-(2,4-dichlorobenzoyl)-4,5-dimethoxyphenyl)acetate (3i). ${ }^{1} \mathrm{H}-\mathrm{NMR}$ (250 MHz): 1.25 (t, 3H, $J=7.2), 3.71$ (s, 3H), 3.95 (s, 3H), 3.96 (s, 2H), 4.15 (q, 2H, $J=7.1), 6.82$ (d, 2H, $J=6.1), 7.26-7.46(\mathrm{~m}, 3 \mathrm{H}) ;{ }^{13} \mathrm{C}-\mathrm{NMR}(62.9 \mathrm{MHz})$ : 194.2, 171.4, 152.4, 147.2, 137.7, 136.6, 132.6, 130.8, 130.5, 130.0, 128.3, 127.1, 115.5, 115.3, 60.8, 56.1, 39.8, 14.2. MS $m / z: 397\left(\mathrm{M}^{+}\right)$.

Ethyl 2-(4,5-dimethoxy-2-(2-(4-methoxyphenyl)acetyl)phenyl)acetate (3k). ${ }^{1} \mathrm{H}-\mathrm{NMR} \quad(250$ MHz): 1.25 (t, $3 \mathrm{H}, J=7), 3.77(\mathrm{~s}, 3 \mathrm{H}), 3.86(\mathrm{~s}, 3 \mathrm{H}), 3.87(\mathrm{~s}, 2 \mathrm{H}), 3.91(\mathrm{~s}, 3 \mathrm{H})$, overlapped with 4.15 (s, 2H), 4.14 (q, 2H, J=7), 6.71 (s, 1H), 6.83-6.87 (m, 2H), 7.14-7.17 (m, 2H), 7.36 (s, 1H); ${ }^{13} \mathrm{C}-\mathrm{NMR}(62.9 \mathrm{MHz})$ : 199.0, 171.6, 158.4, 151.5, 147.1, 130.3, 129.7, 128.7, 127.0, 115.2, 114.1, 113.3, 60.6, 56.1, 55.9, 55.2, 46.8, 40.2, 14.2. $\mathrm{MS} m / z: 372\left(\mathrm{M}^{+}\right)$.

Ethyl 2-(2-(2-naphthoyl)-4,5-dimethoxyphenyl)acetate (3m). ${ }^{1} \mathrm{H}-\mathrm{NMR}(250 \mathrm{MHz}): 1.09$ (t, $3 \mathrm{H}, J=7.1$ ), 3.77 (s, 3H), 3.84 (s, 2H), 3.98 (s, 3H), overlapped with 4.00 (q, 2H, $J=7.1$ ), 6.90 (s, $1 \mathrm{H}), 7.01(\mathrm{~s}, 1 \mathrm{H}), 7.53-7.63(\mathrm{~m}, 2 \mathrm{H}), 7.88-7.94(\mathrm{~m}, 4 \mathrm{H}), 8.23(\mathrm{~s}, 1 \mathrm{H}) ;{ }^{13} \mathrm{C}-\mathrm{NMR}(62.9 \mathrm{MHz})$ : 197.2, 171.5, 150.9, 146.9, 135.6, 135.3, 132.3, 132.2, 130.5, 129.5, 128.4, 128.2, 128.1, 127.7, 126.7, 125.6, 114.5, 113.7, 60.7, 56.1, 55.8, 38.7, 14.1. MS $m / z: 378\left(\mathrm{M}^{+}\right)$.

Ethyl 2-(2-(2,2-diphenylacetyl)-4,5-dimethoxyphenyl)acetate (3n). ${ }^{1} \mathrm{H}-\mathrm{NMR}$ (600 MHz): 1.19 (t, 3H, J=7.1), 3.66 (s, 3H), 3.89 (s, 3H), 3.91 (s, 2H), 4.10 (q, 2H, J=7.1), 5.89 (s, 1H), 6.69 (s, 1H), 7.23-7.33 (m, 11H); ${ }^{13} \mathrm{C}-\mathrm{NMR}$ (150.9 MHz): 199.1, 171.7, 151.5, 147.0, 139.5, 130.3, 129.2, 128.8, 128.7, 127.1, 115.2, 113.7, 61.7, 60.7, 56.0, 55.8, 40.3, 14.2. MS $m / z: 418\left(\mathrm{M}^{+}\right)$.

Methyl 2-(2-(2,2-diphenylacetyl)-5-methoxyphenyl)acetate (3o). ${ }^{1} \mathrm{H}-\mathrm{NMR}$ (600 MHz): 3.59 (s, 3H), $3.80(\mathrm{~s}, 3 \mathrm{H}), 3.94(\mathrm{~s}, 2 \mathrm{H}), 5.96(\mathrm{~s}, 1 \mathrm{H}), 6.74(\mathrm{~d}, 1 \mathrm{H}, J=2.4), 6.76(\mathrm{dd}, 1 \mathrm{H}, J=8.7,2.6)$, 7.23-7.25 (m, 6H), 7.29-7.31 (m, 4H), 7.88 (d, 1H, $J=8.7) ;{ }^{13} \mathrm{C}-\mathrm{NMR}$ (150.9 MHz): 199.1, 171.8, $162.1,139.6,138.5,132.8,129.3,129.2,128.6,127.0,118.8,111.9,60.7,55.4,51.9,40.9$. MS $m / z: 374\left(\mathrm{M}^{+}\right)$.

\section{Preparation of 2-hydroxy-phenyl ethanols (Table 2, 4a-o). Typical procedure}

To solution of $1 \mathrm{mmol}$ of the corresponding ethyl (methyl) 2-acyl-4,5-dimethoxy-phenylacetates 3 in $15 \mathrm{~mL}$ methanol, $\mathrm{NaBH}_{4}(2 \mathrm{mmol}, 0.1 \mathrm{~g})$ was added portionwise. The solution was stirred $30 \mathrm{~min}$ at room temperature, than the solvent was removed under vacuum. Water $(30 \mathrm{~mL})$ was added to the residue and the solution was extracted with $\mathrm{CH}_{2} \mathrm{Cl}_{2}(3 \times 20 \mathrm{~mL})$, then the combined extracts were dried $\left(\mathrm{Na}_{2} \mathrm{SO}_{4}\right)$ and filtered through a short column with silica gel. The products, after evaporation of the solvent, were obtained with $85-90 \%$ yields.

2-(2-(1-Hydroxyethyl)-4,5-dimethoxyphenyl)ethanol (4a). IR $v\left(\mathrm{KBr}, \mathrm{cm}^{-1}\right): 3410,3248$ (OH), 1608 (ArH); ${ }^{1} \mathrm{H}-\mathrm{NMR}$ (600 MHz): 1.48 (dd, 1H, $\left.J=6.4\right), 2.75$ (dt, 1H, J=14.2, 5.1), 2.94 (ddd, $1 \mathrm{H}, J=14.2,8.6,5.8$ ), 3.31 (br s, $2 \mathrm{H}, \mathrm{OH}$ ), 3.69 (ddd, $1 \mathrm{H}, J=10.3,8.7,5.1$ ), 3.82-3.85 (m, $1 \mathrm{H}$ ), overlapped with 3.85 (s, 3H, $\left.\mathrm{OCH}_{3}\right), 3.87$ (s, 3H), 5.05 (q, $\left.1 \mathrm{H}, J=6.4\right), 6.65$ (s, 1H), 6.99 (s, 
1H); ${ }^{13} \mathrm{C}-\mathrm{NMR}(150.9 \mathrm{MHz}): 148.2,147.7,136.0,128.5,112.8,108.6,65.4,63.5,55.9(2$ $\left.\mathrm{OCH}_{3}\right), 34.6,23.3$. MS $m / z: 208\left(\mathrm{M}^{+}-\mathrm{H}_{2} \mathrm{O}\right)$.

1-(2-(2-Hydroxyethyl)-4-methoxyphenyl)ethanol (4b). IR $v\left(\mathrm{KBr}, \mathrm{cm}^{-1}\right): 3276$ (br, OH), 1608 (ArH); ${ }^{1} \mathrm{H}-\mathrm{NMR}(600 \mathrm{MHz}): 1.49$ (d, 3H, $\left.J=6.5\right), 2.80$ (dt, $\left.1 \mathrm{H}, J=14.0,5.1\right), 2.99$ (ddd, $1 \mathrm{H}$, $J=14.2,8.7,5.7$ ), 3.16 (br s, $1 \mathrm{H}, \mathrm{OH}$ ), 3.31 (br s, $1 \mathrm{H}, \mathrm{OH}$ ), 3.74 (ddd, $1 \mathrm{H}, J=10.3,8.8,5.0$ ), 3.78 (s, 3H), 3.87-3.90 (m, $1 \mathrm{H}), 5.05$ (q, $1 \mathrm{H}, J=6.5), 6.71(\mathrm{~d}, 1 \mathrm{H}, J=2.7), 6.78$ (dd, $1 \mathrm{H}, J=8.6,2.7)$, $7.38(\mathrm{~d}, 1 \mathrm{H}, J=8.6) ;{ }^{13} \mathrm{C}-\mathrm{NMR}(150.9 \mathrm{MHz}): 158.9,138.3,135.8,126.7,115.4,112.0,65.2,63.6$, 55.2, 35.0, 23.0. MS $m / z: 178\left(\mathrm{M}^{+}-\mathrm{H}_{2} \mathrm{O}\right)$.

1-(2-(2-Hydroxyethyl)-4,5-dimethoxyphenyl)propan-1-ol (4c). IR $v$ (film, $\left.\mathrm{cm}^{-1}\right): 3260$ (br, OH), 1606 (ArH); ${ }^{1} \mathrm{H}-\mathrm{NMR}$ (250 MHz): 0.93 (t, 3H, $J=7.4$ ), 1.69-1.96 (m, 2H), 2.03 (br s, $1 \mathrm{H}$, $\mathrm{OH}), 2.75$ (dt, $1 \mathrm{H}, J=14.1,5.4), 2.96$ (ddd, $1 \mathrm{H}, J=14.0,8.1,5.9$ ), 3.02 (br s, $1 \mathrm{H}, \mathrm{OH}), 3.70$ (ddd, $1 \mathrm{H}, J=10.3,8.2,5.3), 3.79-3.88(\mathrm{~m}, 1 \mathrm{H})$ overlapped with $3.86\left(\mathrm{~s}, 6 \mathrm{H}, 2 \mathrm{xOCH}_{3}\right), 4.75(\mathrm{dd}, 1 \mathrm{H}$, $J=7.3,6.4), 6.65$ (s, 1H), 6.94 (s, 1H); ${ }^{13} \mathrm{C}-\mathrm{NMR}(62.9 \mathrm{MHz}): 148.2,147.7,135.0,128.8,112.8$, 109.1, 71.3, 63.6, 55.9, 34.7, 30.4, 10.7. MS $m / z: 222\left(\mathrm{M}^{+}-\mathrm{H}_{2} \mathrm{O}\right)$.

1-(2-(2-Hydroxyethyl)-4,5-dimethoxyphenyl)-3-methylbutan-1-ol (4d). IR $v\left(\mathrm{KBr}, \mathrm{cm}^{-1}\right)$ : 3333 (br, OH), 1608 (ArH); ${ }^{1} \mathrm{H}-\mathrm{NMR}(250 \mathrm{MHz}): 0.95$ (dd, 6H, $\left.J=6.4,2.8\right), 1.43-1.54(\mathrm{~m}, 1 \mathrm{H})$, 1.69-1.84 (m, 1H), 2.02 (br s, 1H, OH), 2.75 (dt, 1H, J=14.2, 5.2), 2.82 (br s, 1H, OH), 2.97 (ddd, $1 \mathrm{H}, J=14.1,8.3,5.8), 3.71$ (ddd, $1 \mathrm{H}, J=10.3,8.4,5.1$ ), overlapped with 3.85 (s, $3 \mathrm{H}, \mathrm{OCH}_{3}$ ), 3.87 (s, 3H), 4.93 (dd, 1H, J=8.4, 5.0), 6.65 (s, 1H), 6.95 (s, 1H); ${ }^{13} \mathrm{C}-\mathrm{NMR}(62.9 \mathrm{MHz}): 148.1$, 147.7, 135.6, 128.4, 112.8, 109.1, 67.8, 63.6, 55.9, 46.6, 34.6, 25.1, 23.4, 22.3. MS m/z: 250 $\left(\mathrm{M}^{+}-\mathrm{H}_{2} \mathrm{O}\right)$.

1-(2-(2-Hydroxyethyl)-4,5-dimethoxyphenyl)hexadecan-1-ol (4e). IR $\vee\left(\mathrm{KBr}, \mathrm{cm}^{-1}\right): 3517$, $3357(\mathrm{OH}), 1607$ (ArH); ${ }^{1} \mathrm{H}-\mathrm{NMR}(600 \mathrm{MHz}): 0.88$ (t, 3H, $\left.J=7.1\right), 1.25$ (br s, 26H), 1.28-1.39 (m, 1H), 1.42-1.48 (m, 1H), 1.68-1.73 (m, 1H), $1.80($ br s, $1 \mathrm{H}, \mathrm{OH}), 1.82-1.88(\mathrm{~m}, 1 \mathrm{H}), 2.71(\mathrm{br}$ s, $1 \mathrm{H}, \mathrm{OH}$ ), 2.77 (dt, $1 \mathrm{H}, J=14.1,5.3$ ), 2.97 (ddd, $1 \mathrm{H}, J=14.1,8.3,5.8$ ), 3.73 (ddd, $1 \mathrm{H}, J=10.3$, 8.3, 5.2), 3.84-3.89 $(\mathrm{m}, 1 \mathrm{H})$, overlapped with $3.86\left(\mathrm{~s}, 3 \mathrm{H}, \mathrm{OCH}_{3}\right)$ and $3.87\left(\mathrm{~s}, 3 \mathrm{H}, \mathrm{OCH}_{3}\right), 4.84$ (dd, 1H, J=7.9, 5.8), $6.65(\mathrm{~s}, 1 \mathrm{H}), 6.95(\mathrm{~s}, 1 \mathrm{H}) ;{ }^{13} \mathrm{C}-\mathrm{NMR}(150.9 \mathrm{MHz}): 148.2,147.8,135.4$, 128.6, 112.8, 109.1, 69.9, 63.7, 55.9, 37.6, 34.7, 31.9, 29.7, 29.4, 26.4, 22.7, 14.2. MS $m / z: 404$ $\left(\mathrm{M}^{+}-\mathrm{H}_{2} \mathrm{O}\right)$.

2-(2-(Hydroxy(phenyl)methyl)-4,5-dimethoxyphenyl)ethanol (4f). IR $v\left(\mathrm{KBr}, \mathrm{cm}^{-1}\right): 3495$, 3317 (OH), 1608 (ArH); ${ }^{1} \mathrm{H}-\mathrm{NMR}(600 \mathrm{MHz}): 2.73$ (dt, $\left.1 \mathrm{H}, J=14.3,5.3\right) 2.92$ (ddd, $1 \mathrm{H}, J=13.8$, 8.2, 5.3), $3.69(\mathrm{~s}, 3 \mathrm{H}), 3.64-3.68(\mathrm{~m}, 1 \mathrm{H})$, overlapped with $3.69\left(\mathrm{~s}, 3 \mathrm{H}, \mathrm{OCH}_{3}\right), 3.77-3.80(\mathrm{~m}$, $1 \mathrm{H}), 3.84(\mathrm{~s}, 3 \mathrm{H}), 5.96(\mathrm{~s}, 1 \mathrm{H}), 6.65(\mathrm{~s}, 1 \mathrm{H}), 6.66(\mathrm{~s}, 1 \mathrm{H}), 7.23-7.33(\mathrm{~m}, 5 \mathrm{H}) ;{ }^{13} \mathrm{C}-\mathrm{NMR}(150.9$ MHz): 148.5, 147.4, 134.9, 129.6, 128.2, 127.1, 126.5, 112.8, 111.5, 72.2, 63.5, 55.9, 55.8, 34.7. MS $m / z: 270\left(\mathrm{M}^{+}-\mathrm{H}_{2} \mathrm{O}\right)$.

2-(2-((3,4-Dimethoxyphenyl)(hydroxyl)methyl)-4,5-dimethoxyphenyl)ethanol (4g). IR $v$ $\left(\mathrm{KBr}, \mathrm{cm}^{-1}\right): 3536,3485(\mathrm{OH}), 1607(\mathrm{ArH}) ;{ }^{1} \mathrm{H}-\mathrm{NMR}(600 \mathrm{MHz}): 1.85$ (br s, 1H, OH), 2.77 (dt, $1 \mathrm{H}, J=14.2,5.1), 2.81$ (br s, $1 \mathrm{H}, \mathrm{OH}), 2.96$ (ddd, $1 \mathrm{H}, J=13.9,8.4,5.3), 3.69-3.74$ (m, $1 \mathrm{H})$, overlapped with $3.72\left(\mathrm{~s}, 3 \mathrm{H}, \mathrm{OCH}_{3}\right), 3.82-3.88(\mathrm{~m}, 1 \mathrm{H})$, overlapped with $3.83\left(\mathrm{~s}, 3 \mathrm{H}, \mathrm{OCH}_{3}\right)$ and $3.86\left(\mathrm{~s}, 3 \mathrm{H}, \mathrm{OCH}_{3}\right)$ and $3.87\left(\mathrm{~s}, 3 \mathrm{H}, \mathrm{OCH}_{3}\right), 5.95(\mathrm{~s}, 1 \mathrm{H}), 6.69(\mathrm{~s}, 1 \mathrm{H}), 6.7(\mathrm{~s}, 1 \mathrm{H}), 6.81-6.83(\mathrm{~m}$, 
$3 \mathrm{H}) ;{ }^{13} \mathrm{C}-\mathrm{NMR}$ (150.9 MHz): 148.8, 148.5, 148.0, 147.4, 136.0, 135.0, 129.5, 118.8, 112.8, 111.3, 110.8, 109.9, 71.9, 63.6, 56.1, 55.9, 55.8, 34.7. $\mathrm{MS} m / z: 330\left(\mathrm{M}^{+}-\mathrm{H}_{2} \mathrm{O}\right)$.

2-(2-(Hydroxy(3,4,5-trimethoxyphenyl)methyl)-4,5-dimethoxyphenyl)ethanol (4h). IR $v$ $\left(\mathrm{KBr}, \mathrm{cm}^{-1}\right):$ 3495, $3322(\mathrm{OH}), 1592(\mathrm{ArH}) ;{ }^{1} \mathrm{H}-\mathrm{NMR}(600 \mathrm{MHz}): 1.72$ (br s, 1H, OH), 2.40 (br s, $1 \mathrm{H}, \mathrm{OH}), 2.72$ (dt, $1 \mathrm{H}, J=16.1,3.6), 3.05$ (ddd, $1 \mathrm{H}, J=15.4,8.8,6.0), 3.69$ (s, 3H), 3.80-3.85 $(\mathrm{m}, 1 \mathrm{H})$ overlapped with $3.82\left(\mathrm{~s}, 6 \mathrm{H}, 2 \mathrm{xOCH}_{3}\right)$ and $3.84\left(\mathrm{~s}, 3 \mathrm{H}, \mathrm{OCH}_{3}\right), 3.88-3.92(\mathrm{~m}, 1 \mathrm{H})$ overlapped with $3.91\left(\mathrm{~s}, 3 \mathrm{H}, \mathrm{OCH}_{3}\right), 5.60(\mathrm{~s}, 1 \mathrm{H}), 6.29(\mathrm{~s}, 1 \mathrm{H}), 6.53(\mathrm{~s}, 2 \mathrm{H}), 6.66(\mathrm{~s}, 1 \mathrm{H}) ;{ }^{13} \mathrm{C}-$ NMR (150.9 MHz): 153.2, 147.9, 147.2, 137.8, 128.6, 126.1, 111.2, 109.7, 106.0, 103.6, 79.6, 60.9, 56.2, 56.1, 56.0, 55.9, 28.3. MS $m / z: 360\left(\mathrm{M}^{+}-\mathrm{H}_{2} \mathrm{O}\right)$.

2-(2-((2,4-Dichlorophenyl)(hydroxyl)methyl)-4,5-dimethoxyphenyl)ethanol (4i). IR $v(\mathrm{KBr}$, $\mathrm{cm}^{-1}$ ): 3506, $3347(\mathrm{OH}), 1608$ (ArH); ${ }^{1} \mathrm{H}-\mathrm{NMR}$ (600 MHz): 2.78 (dt, 1H, J=14.4, 3.9), 2.82 (br s, $1 \mathrm{H}, \mathrm{OH}), 3.20$ (ddd, $1 \mathrm{H}, J=14.8,10.2,5.0), 3.62$ (s, 3H), 3.74 (td, $1 \mathrm{H}, J=10.1,3.7), 3.85$ (s, 3H), 3.99 (tt, 1H, J=8.3, 4.1), 4.54 (br s, 1H, OH), 6.15 (s, 1H), 6.32 (s, 1H), 6.69 (s, 1H), 7.32 (dd, $1 \mathrm{H}, J=8.3,2.1), 7.34$ (d, 1H, $J=2.1), 7.70$ (d, 1H, $J=8.3$ ); ${ }^{13} \mathrm{C}-\mathrm{NMR}$ (150.9 MHz): 148.8, 147.6, $139.2,133.5,133.0,130.2,129.3,128.9,127.0,112.7,110.3,68.5,63.6,55.9,55.8,34.5$. MS $m / z: 338\left(\mathrm{M}^{+}-\mathrm{H}_{2} \mathrm{O}\right)$.

1-(2-(2-Hydroxyethyl)-4,5-dimethoxyphenyl)-2-phenylethanol (4j). IR $v\left(\mathrm{KBr}, \mathrm{cm}^{-1}\right): 3305$ (br, OH), 1608 (ArH); ${ }^{1} \mathrm{H}-\mathrm{NMR}$ (600 MHz): 2.21 (s, 1H, OH), 2.69 (dt, 1H, J=14.1, 5.8), 2.82 (ddd, 1H, $J=13.9,7.7,6.1$ ), 2.88 (br s, 1H, OH), 3.03 (dq, 1H, J=13.6, 6.7), 3.64 (ddd, 1H, $J=10.4,7.7,5.8$ ), 3.71 (dt, $1 \mathrm{H}, J=11.6,4.6), 3.85$ (s, 3H), 3.86 (s, 3H), 5.06 (dd, 1H, $J=8.1,5.3$ ), $6.62(\mathrm{~s}, 1 \mathrm{H}), 7.00(\mathrm{~s}, 1 \mathrm{H}), 7.17(\mathrm{~d}, 1 \mathrm{H}, J=6.9), 7.23-7.19(\mathrm{~m}, 1 \mathrm{H}), 7.27(\mathrm{dd}, 1 \mathrm{H}, J=10.6,4.0)$; ${ }^{13} \mathrm{C}-\mathrm{NMR}$ (150.9 MHz): 148.2, 147.7, 138.4, 134.3, 129.5, 128.5, 128.2, 126.5, 112.7, 109.3, 71.2, 63.6, 56.0, 55.9, 44.7, 34.6. MS $m / z: 284\left(\mathrm{M}^{+}-\mathrm{H}_{2} \mathrm{O}\right)$.

1-(2-(2-Hydroxyethyl)-4,5-dimethoxyphenyl)-2-(4-methoxyphenyl)ethanol (4k). IR $v$ ( $\mathrm{KBr}$, $\mathrm{cm}^{-1}$ ): 3312 (br, OH), 1610 (ArH); ${ }^{1} \mathrm{H}-\mathrm{NMR}$ (250 MHz): 2.51 (br s, 2H, OH), 2.68 (dt, 1H, $J=11.9,5.7), 2.82$ (dt, $1 \mathrm{H}, J=13.8,6.8), 2.96$ (dd, $2 \mathrm{H}, J=6.5,2.8), 3.59-3.68$ (m, 1H), 3.71 (dd, $1 \mathrm{H}, J=10.1,4.2), 3.76(\mathrm{~s}, 3 \mathrm{H}), 3.85$ (s, 3H), 3.86 (s, 3H), $5.02(\mathrm{dd}, 1 \mathrm{H}, J=7.3,6.0), 6.62$ (s, 1H), $6.80(\mathrm{~d}, 2 \mathrm{H}, J=8.6), 7.03(\mathrm{~d}, 2 \mathrm{H}, J=8.6), 7.10(\mathrm{~s}, 1 \mathrm{H}) ;{ }^{13} \mathrm{C}-\mathrm{NMR}(62.9 \mathrm{MHz}): 158.2,148.1$, $147.6,134.3,130.4,130.3,128.2,113.8,112.6,109.2,71.2,63.5,55.9,55.8,55.2,43.7$, 34.6. MS $m / z: 314\left(\mathrm{M}^{+}-\mathrm{H}_{2} \mathrm{O}\right)$.

2-(3,4-Dimethoxyphenyl)-1-(2-(2-hydroxyethyl)-4,5-dimethoxyphenyl)ethanol (4I). IR $v$ (KBr, cm ${ }^{-1}$ ): 3348 (br, OH), 1605 (ArH); ${ }^{1} \mathrm{H}-\mathrm{NMR}$ (600 MHz): 2.20 (br s, 1H, OH), 2.69 (dt, 1H, $J=14.1,5.7$ ), 2.80-2.85 (ddd, $1 \mathrm{H}, J=14.1,7.5,6.2$ ), 2.86 (br s, 1H, OH), 2.95 (dd, $1 \mathrm{H}, J=13.7$, 5.2), 3.01 (dd, $1 \mathrm{H}, J=13.7,8.1$ ), 3.67-3.69 (m, 1H), 3.75 (dd, $1 \mathrm{H}, J=11.1,5.3), 3.80$ (s, 3H), 3.84 $(\mathrm{s}, 3 \mathrm{H}), 3.86(\mathrm{~s}, 3 \mathrm{H}), 3.88(\mathrm{~s}, 3 \mathrm{H}), 5.05(\mathrm{dd}, 1 \mathrm{H}, J=8.1,5.2), 6.63(\mathrm{~s}, 1 \mathrm{H}), 6.66(\mathrm{~d}, 1 \mathrm{H}, J=1.7)$, $6.74(\mathrm{dd}, 1 \mathrm{H}, J=8.1,1.8), 6.78(\mathrm{~d}, 1 \mathrm{H}, J=8.1), 7.02$ (s, $1 \mathrm{H}) ;{ }^{13} \mathrm{C}-\mathrm{NMR}(150.9 \mathrm{MHz}): 148.8,148.3$, $147.8,147.7,134.4,130.9,128.4,121.4,112.7,111.2,109.3,71.3,63.6,56.0,55.9,55.8,44.3$, 34.7. $\mathrm{MS} m / z: 344\left(\mathrm{M}^{+}-\mathrm{H}_{2} \mathrm{O}\right)$.

2-(2-(Hydroxy-(naphth-2-yl)-methyl)-4,5-dimethoxyphenyl)ethanol (4m). IR $v\left(\mathrm{KBr}, \mathrm{cm}^{-1}\right)$ : 3263 (br, OH), 1606 (ArH); ${ }^{1} \mathrm{H}-\mathrm{NMR}$ (600 MHz): 2.76 (dt, 1H, J=14.3, 5.1), 2.95 (ddd, 1H, 
$J=13.8,8.4,5.1$ ), 3.09 (br s, $1 \mathrm{H}, \mathrm{OH}), 3.62$ (s, 3H), 3.69 (ddd, $1 \mathrm{H}, J=10.0,8.5,4.6), 3.82$ (s, 3H, $\left.\mathrm{OCH}_{3}\right)$, overlapped with 3.80-3.84 (m, 1H), $4.42($ br s, $1 \mathrm{H}, \mathrm{OH}), 6.10(\mathrm{~s}, 1 \mathrm{H}), 6.65(\mathrm{~s}, 1 \mathrm{H}), 6.66$ (s, 1H), $7.30(\mathrm{dd}, 1 \mathrm{H}, J=6.7,1.7), 7.44-7.46(\mathrm{~m}, 2 \mathrm{H}), 7.74(\mathrm{~d}, 1 \mathrm{H}, J=8.5), 7.77-7.81(\mathrm{~m}, 2 \mathrm{H})$, 7.90 (s, 1H); ${ }^{13} \mathrm{C}-\mathrm{NMR}$ (150.9 MHz): 148.6, 147.5, 140.8, 134.7, 133.2, 132.6, 129.8, 128.1, $127.9,127.6,126.1,125.8,125.0,124.7,112.8,111.7,72.2,63.6,55.9,55.8,34.7$. MS m/z: 320 $\left(\mathrm{M}^{+}-\mathrm{H}_{2} \mathrm{O}\right)$.

1-(2-(2-Hydroxyethyl)-4,5-dimethoxyphenyl)-2,2-diphenylethanol (4n). IR $v\left(\mathrm{KBr}, \mathrm{cm}^{-1}\right)$ : 3492, 3359 (OH), 1601 (ArH); ${ }^{1} \mathrm{H}-\mathrm{NMR}$ (600 MHz): 1.68 (br s, 1H, OH), 2.11 (br s, 1H, OH), 2.59 (dt, 1H, $J=14.1,5.6$ ), 2.83 (ddd, $1 \mathrm{H}, J=13.8,7.7,5.9), 3.61-3.65$ (m, 1H), 3.67-3.71 (m, $1 \mathrm{H}), 3.74(\mathrm{~s}, 3 \mathrm{H}), 3.81(\mathrm{~s}, 3 \mathrm{H}), 4.36(\mathrm{~d}, 1 \mathrm{H}, J=9.2), 5.61(\mathrm{~d}, 1 \mathrm{H}, J=9.2), 6.53(\mathrm{~s}, 1 \mathrm{H}), 6.82(\mathrm{~s}$, 1H), 7.04-7.12 (m, 5H), $7.25(\mathrm{t}, 1 \mathrm{H}, J=7.4), 7.35(\mathrm{t}, 2 \mathrm{H}, J=7.7), 7.44(\mathrm{~d}, 2 \mathrm{H}, J=7.8) ;{ }^{13} \mathrm{C}-\mathrm{NMR}$ (150.9 MHz): 148.2, 147.3, 141.6, 141.2, 132.6, 129.1, 128.5, 128.2, 126.9, 126.3, 112.3, 110.3, 72.4, 63.6, 58.8, 55.8, 55.7, 34.6. MS m/z: $360\left(\mathrm{M}^{+}-\mathrm{H}_{2} \mathrm{O}\right)$.

1-(2-(2-Hydroxyethyl)-4-methoxyphenyl)-2,2-diphenylethanol (4o). IR $v\left(\mathrm{KBr}, \mathrm{cm}^{-1}\right): 3496$, $3360(\mathrm{OH}), 1599$ (ArH); ${ }^{1} \mathrm{H}-\mathrm{NMR}(600 \mathrm{MHz}): 2.07$ (br s, 1H, OH), 2.15 (br s, 1H, OH), 2.65$2.69(\mathrm{~m}, 1 \mathrm{H}), 2.88-2.93(\mathrm{~m}, 1 \mathrm{H}), 3.61-3.65(\mathrm{~m}, 1 \mathrm{H}), 3.67-3.71(\mathrm{~m}, 1 \mathrm{H}), 3.80(\mathrm{~s}, 3 \mathrm{H}), 4.41(\mathrm{~d}, 1 \mathrm{H}$, $J=10), 5.59$ (d, 1H, $J=10), 6.74$ (d, 1H, $J=2.4), 6.76$ (dd, $1 \mathrm{H}, J=8.7,2.6), 7.20-7.26$ (m, 7H), 7.30 (t, 3H, J=7.6), 7.88 (d, 1H, J=8.7); ${ }^{13} \mathrm{C}-\mathrm{NMR}$ (150.9 MHz): 162.1, 139.6, 138.5, 132.8, 129.3, $129.2,128.8,128.6,127.0,126.3,118.8,111.9,60.8,55.4,51.8,40.9,29.7$. MS m/z: $330\left(\mathrm{M}^{+}-\right.$ $\left.\mathrm{H}_{2} \mathrm{O}\right)$.

\section{Synthesis of 1-substituted isochromanes 5. Typical procedure}

A catalytic amount of $p$-toluenesulfonic acid monohydrate (PTSA) was added to a solution of 1 mmol of the corresponding 2-hydroxy-4,5-dimethoxyphenylethanols $\mathbf{4}$ in dichloromethane. The solution was stirred $30 \mathrm{~min}$ at room temperature. Then the solution was filtered through a short column of silica gel. The products, after evaporation of the solvent, were obtained with 88-90\% yield.

6,7-Dimethoxy-1-methylisochroman (5a). ${ }^{1} \mathrm{H}-\mathrm{NMR}(250 \mathrm{MHz}): 1.51$ (d, 3H, $\left.J=6.5\right), 2.56-2.64$ $(\mathrm{m}, 1 \mathrm{H}), 2.91-2.95(\mathrm{~m}, 1 \mathrm{H}), 3.72-3.82(\mathrm{~m}, 1 \mathrm{H}), 3.85(\mathrm{~s}, 3 \mathrm{H}), 3.86(\mathrm{~s}, 3 \mathrm{H}), 4.10-4.18(\mathrm{~m}, 1 \mathrm{H})$, 4.79 (q, 1H, J=6.5), 6.56 (s, 1H), 6.60 (s, 1H); ${ }^{13} \mathrm{C}-\mathrm{NMR}(62.9 \mathrm{MHz}): 148.8,146.9,128.9,128.2$, 111.6, 107.6, 79.7, 62.3, 55.9, 55.8, 28.9, 14.6. MS m/z: $208(\mathrm{M}+)$; Anal. calcd. for $\mathrm{C}_{12} \mathrm{H}_{16} \mathrm{O}_{3}$ : C 69.21, H 7.74. Found: C 69.33, H 7.86.

6-Methoxy-1-methylisochroman (5b). ${ }^{1} \mathrm{H}-\mathrm{NMR}(250 \mathrm{MHz}): 1.51$ (d, 3H, J=6.5), 2.56-2.64 (m, $1 \mathrm{H}), 2.81-2.95(\mathrm{~m}, 1 \mathrm{H}), 3.72-3.84(\mathrm{~m}, 1 \mathrm{H}), 3.85(\mathrm{~s}, 3 \mathrm{H}), 4.78(\mathrm{q}, 1 \mathrm{H}, J=6.5), 6.61-7.12(\mathrm{~m}, 3 \mathrm{H})$ $3.85(\mathrm{~s}, 3 \mathrm{H}), 3.86(\mathrm{~s}, 3 \mathrm{H}), 4.10-4.18(\mathrm{~m}, 1 \mathrm{H}), 4.79(\mathrm{q}, 1 \mathrm{H}, J=6.5), 6.56(\mathrm{~s}, 1 \mathrm{H}), 6.60(\mathrm{~s}, 1 \mathrm{H}) ;{ }^{13} \mathrm{C}-$ NMR (62.9 MHz): 159.6, 136.6, 128.3, 127.2, 113.4, 110.9, 79.4, 62.3, 55.8, 28.9, 14.5. MS m/z: $178(\mathrm{M}+)$; Anal. Calcd. for $\mathrm{C}_{11} \mathrm{H}_{14} \mathrm{O}_{2}$ : C 74.13, H 7.92. Found: C 74.23, H 7.80.

6,7-Dimethoxy-1-ethylisochroman (5c). ${ }^{1} \mathrm{H}-\mathrm{NMR}(250 \mathrm{MHz}): 1.01(\mathrm{t}, 3 \mathrm{H}, J=7.3), 1.82$ (dd, $1 \mathrm{H}, J=14.9,7.3$ ), 1.97 (ddd, $1 \mathrm{H}, J=14.4,7.4,3.3$ ), 2.61 (dt, 1H, $J=15.9,3.6$ ), 2.93 (ddd, 1H, $J=14.9,9.4,5.3), 3.77$ (ddd, $1 \mathrm{H}, J=11.2,9.6,3.8), 3.87$ (s, 3H), 3.88 (s, 3H), 4.14 (ddd, 1H, 
$J=11.2,5.3,3.7), 4.66(\mathrm{dd}, 1 \mathrm{H}, J=7.8,2.5), 6.58(\mathrm{~s}, 1 \mathrm{H}), 6.62(\mathrm{~s}, 1 \mathrm{H}) ;{ }^{13} \mathrm{C}-\mathrm{NMR}(62.9 \mathrm{MHz})$ : 147.8, 147.3, 130.1, 126.1, 111.4, 107.8, 63.2, 55.9, 55.8, 28.8, 28.7, 9.5. MS m/z: $222(\mathrm{M}+)$; Anal. Calcd. for $\mathrm{C}_{13} \mathrm{H}_{18} \mathrm{O}_{3}: \mathrm{C} 70.24, \mathrm{H}$ 8.16. Found: C 70.36, H 8.28.

6,7-Dimethoxy-1-isobutylisochroman (5d). ${ }^{1} \mathrm{H}-\mathrm{NMR}(250 \mathrm{MHz}): 0.98$ (dd, $\left.6 \mathrm{H}, J=15.6,6.6\right)$, 1.53 (ddd, $1 \mathrm{H}, J=14.2,10.0,2.9$ ), 1.75 (ddd, $1 \mathrm{H}, J=14.2,10.3,3.8$ ), 1.91-2.04 (m, 1H), 2.62 (dt, $1 \mathrm{H}, J=16.0,4.3), 2.74-2.88(\mathrm{~m}, 1 \mathrm{H}), 2.88-3.01(\mathrm{~m}, 1 \mathrm{H}), 3.72$ (ddd, $1 \mathrm{H}, J=12.4,7.5,4.1), 3.84$ (s, $3 \mathrm{H}), 3.85(\mathrm{~s}, 3 \mathrm{H}), 4.08(\mathrm{dt}, 1 \mathrm{H}, J=11.2,4.8), 4.71(\mathrm{dd}, 1 \mathrm{H}, J=9.5,1.8), 6.51(\mathrm{~s}, 1 \mathrm{H}), 6.58(\mathrm{~s}, 1 \mathrm{H})$; ${ }^{13} \mathrm{C}-\mathrm{NMR}(62.9 \mathrm{MHz}): 147.4,147.3,131.0,125.8,111.5,107.9,73.6,62.8,56.0,55.9,45.3$, 28.6, 24.5, 24.0, 21.5. MS m/z: $250(\mathrm{M}+)$; Anal. Calcd. for $\mathrm{C}_{15} \mathrm{H}_{22} \mathrm{O}_{3}$ : C 71.97, H 8.86. Found: $\mathrm{C}$ $72.08, \mathrm{H} 8.98$.

6,7-Dimethoxy-1-pentadecyl-isochroman (5e). ${ }^{1} \mathrm{H}-\mathrm{NMR}(600 \mathrm{MHz}): 0.88$ (t, 3H, $\left.J=7.0\right), 1.25$ $1.31(\mathrm{~m}, 23 \mathrm{H}), 1.32-1.39(\mathrm{~m}, 1 \mathrm{H}), 1.43-1.51(\mathrm{~m}, 2 \mathrm{H}), 1.73-1.79(\mathrm{~m}, 1 \mathrm{H}), 1.85-1.89(\mathrm{~m}, 1 \mathrm{H}), 2.60$ (dt, $1 \mathrm{H}, J=15.8,3.7$ ), 2.90 (ddd, $1 \mathrm{H}, J=15.1,9.4,5.3$ ), 3.74 (ddd, $1 \mathrm{H}, J=11.1,9.5,3.8$ ), 3.85 (s, $3 \mathrm{H}), 3.86$ (s, 3H), 4.11 (ddd, $1 \mathrm{H}, J=11.2,5.2,3.8), 4.67$ (dd, $1 \mathrm{H}, J=8.3,2.2), 6.55$ (s, 1H), 6.59 (s, $1 \mathrm{H}) ;{ }^{13} \mathrm{C}-\mathrm{NMR}(150.9 \mathrm{MHz}): 147.4,130.5,126.0,111.4,107.9,75.6,63.2,56.0,55.9,36.2$, 32.0, 29.8, 29.7, 25.4, 22.7, 14.2. MS m/z: $404(\mathrm{M}+)$; Anal. Calcd. for $\mathrm{C}_{26} \mathrm{H}_{44} \mathrm{O}_{3}$ : C 77.18, $\mathrm{H}$ 10.96. Found: C 77.28, H 11.08 .

6,7-Dimethoxy-1-(3,4,5-trimethoxyphenyl)isochroman (5h). ${ }^{1} \mathrm{H}-\mathrm{NMR}(600 \mathrm{MHz}): 2.72$ (dt, $1 \mathrm{H}, J=16.0,3.8), 3.06$ (ddd, $1 \mathrm{H}, J=15.2,9.2,5.7), 3.70$ (s, 3H), 3.82 (s, 6H), 3.85 (s, 3H), 3.89 (s, $3 \mathrm{H}, \mathrm{OCH}_{3}$ ), overlapped with 3.88-3.91 (m, $\left.1 \mathrm{H}\right), 4.19$ (ddd, $\left.1 \mathrm{H}, J=11.3,5.4,3.9\right), 5.60(\mathrm{~s}, 1 \mathrm{H})$, $6.29(\mathrm{~s}, 1 \mathrm{H}), 6.53(\mathrm{~s}, 2 \mathrm{H}), 6.66(\mathrm{~s}, 1 \mathrm{H}) ;{ }^{13} \mathrm{C}-\mathrm{NMR}$ (150.9 MHz): 153.2, 148.0, 147.2, 137.8, 137.7, 128.6, 126.1, 111.2, 109.7, 106.0, 79.6, 64.0, 60.8, 56.1, 56.0, 55.9, 28.4. MS $m / z: 360$ $(\mathrm{M}+)$; Anal. Calcd. for $\mathrm{C}_{20} \mathrm{H}_{24} \mathrm{O}_{6}$ : C 66.65, H 6.71. Found: C 66.78, H 6.82.

6,7-Dimethoxy-1-(2,4-dichlorophenyl)isochroman (5i). ${ }^{1} \mathrm{H}-\mathrm{NMR}(250 \mathrm{MHz}): 2.74$ (dt, 1H, $J=15.9,3.7), 3.02$ (ddd, $1 \mathrm{H}, J=8.4,7.5,5.5), 3.88(\mathrm{~s}, 3 \mathrm{H}), 3.92\left(\mathrm{~s}, 3 \mathrm{H}, \mathrm{OCH}_{3}\right)$, overlapped with 3.90-3.96 (m, 1H), 4.11 (dt, 1H, $J=11.2,4.9), 6.14(\mathrm{~s}, 1 \mathrm{H}), 6.21(\mathrm{~s}, 1 \mathrm{H}), 6.65(\mathrm{~s}, 1 \mathrm{H}), 7.09(\mathrm{~d}$, $1 \mathrm{H}, J=8.4), 7.18$ (dd, $2 \mathrm{H}, J=8.4,2.0), 7.46$ (d, $1 \mathrm{H}, J=2.0) ;{ }^{13} \mathrm{C}-\mathrm{NMR}(62.9 \mathrm{MHz}): 148.1,147.5$, 138.5, 134.9, 134.3, 131.6, 129.3, 127.7, 127.3, 126.2, 111.3, 109.0, 74.4, 63.7, 55.9, 55.8, 28.2. MS m/z: $339(\mathrm{M}+)$; Anal. Calcd. for $\mathrm{C}_{17} \mathrm{H}_{16} \mathrm{Cl}_{2} \mathrm{O}_{3}$ : C 60.19, H 4.75. Found: C 60.30, H 4.87.

6,7-Dimethoxy-1-benzylisochroman (5j). ${ }^{1} \mathrm{H}-\mathrm{NMR}(250 \mathrm{MHz}): 2.64$ (dt, $\left.2 \mathrm{H}, J=15.8,4.2\right), 2.81$ (ddd, $1 \mathrm{H}, J=13.6,8.4,4.8$ ), 3.10 (dd, $1 \mathrm{H}, J=14.3,7.9$ ), 3.15 (dd, $1 \mathrm{H}, J=14.2,4.8$ ), 3.72 (m, $1 \mathrm{H}$ ), overlapped with $3.76\left(\mathrm{~s}, 3 \mathrm{H}, \mathrm{OCH}_{3}\right), 3.85(\mathrm{~s}, 3 \mathrm{H}), 4.15(\mathrm{dt}, 1 \mathrm{H}, J=11.4,4.8), 4.96(\mathrm{dd}, 1 \mathrm{H}$, $J=11.4,4.8), 6.47$ (s, $1 \mathrm{H}), 6.59$ (s, $1 \mathrm{H}), 7.24-7.31$ (m, 5H); ${ }^{13} \mathrm{C}-\mathrm{NMR}$ (62.9 MHz): 147.6, 147.2, 138.8, 130.1, 129.6, 128.3, 126.3, 111.5, 108.3, 76.3, 62.8, 56.2, 55.9, 42.8, 28.6. MS $m / z: 284$ $(\mathrm{M}+)$; Anal. Calcd. for $\mathrm{C}_{18} \mathrm{H}_{20} \mathrm{O}_{3}$ : C 76.03, H 7.09. Found: C 76.14, H 7.21.

6,7-Dimethoxy-1-(4-methoxybenzyl)isochroman (5k). ${ }^{1} \mathrm{H}-\mathrm{NMR}(250 \mathrm{MHz}): 2.61(\mathrm{dt}, 1 \mathrm{H}$, $J=15.8,4.2), 2.80$ (ddd, $1 \mathrm{H}, J=13.6,8.4,4.9$ ), 3.01 (dd, $1 \mathrm{H}, J=14.3,7.9), 3.11$ (dd, $1 \mathrm{H}, J=14.2$, 4.8), 3.68-3.76 (m, $1 \mathrm{H}), 3.78$ (s, 3H), $3.79(\mathrm{~s}, 3 \mathrm{H}), 3.86(\mathrm{~s}, 3 \mathrm{H}), 4.14$ (dt, $1 \mathrm{H}, J=11.3,4.8), 4.91$ (dd, 1H, J=7.5, 5.0), 6.49 (s, 1H), $6.59(\mathrm{~s}, 1 \mathrm{H}), 6.82(\mathrm{~s}, 1 \mathrm{H}), 6.86(\mathrm{~s}, 1 \mathrm{H}), 7.17(\mathrm{~s}, 1 \mathrm{H}), 7.20(\mathrm{~s}$, $1 \mathrm{H}) ;{ }^{13} \mathrm{C}-\mathrm{NMR}$ (62.9 MHz): 158.1, 147.5, 147.1, 130.8, 130.5, 129.6, 126.2, 113.7, 111.4, 108.3, 
76.4, 62.9, 55.9, 55.2, 41.9, 28.7. MS m/z: $314(\mathrm{M}+)$; Anal. Calcd. for $\mathrm{C}_{19} \mathrm{H}_{22} \mathrm{O}_{4}$ : C 72.59, $\mathrm{H}$ 7.05. Found: C 72.71, H 7.17.

6,7-Dimethoxy-1-(naphth-2-yl)isochroman (5m). ${ }^{1} \mathrm{H}-\mathrm{NMR}(600 \mathrm{MHz}): 2.77$ (dt, 1H, J=16.1, 4.1), 3.08 (ddd, 1H, $J=14.9,8.9,5.4$ ), 3.60 (s, 3H), 3.89 (s, 3H), 3.93 (ddd, 1H, $J=11.3,9.0,4.1$ ), 4.17 (ddd, 1H, $J=11.2,5.2,4.5), 5.84(\mathrm{~s}, 1 \mathrm{H}), 6.26(\mathrm{~s}, 1 \mathrm{H}), 6.69$ (s, 1H), 7.41 (dd, 1H, J=8.5, 1.6), $7.46(\mathrm{t}, 1 \mathrm{H}, J=3.4), 7.48(\mathrm{t}, 1 \mathrm{H}, J=3.4), 7.76(\mathrm{~s}, 1 \mathrm{H}), 7.81(\mathrm{~s}, 1 \mathrm{H}), 7.82-7.84(\mathrm{~m}, 2 \mathrm{H}) ;{ }^{13} \mathrm{C}-$ NMR (150.9 MHz): 147.9, 147.3, 139.5, 133.3, 133.1, 128.8, 128.4, 128.2, 128.1, 127.7, 126.6, 126.2, 126.1, 126.0, 111.2, 109.7, 79.3, 63.6, 55.9, 55.8, 28.4. MS m/z: $320(\mathrm{M}+)$; Anal. Calcd. for $\mathrm{C}_{21} \mathrm{H}_{20} \mathrm{O}_{3}$ : C 78.73, $\mathrm{H}$ 6.29. Found: C 78.85, H 6.40.

6,7-Dimethoxy-1-benzhydrylisochroman (5n). ${ }^{1} \mathrm{H}-\mathrm{NMR}(600 \mathrm{MHz}): 2.55$ (dt, $1 \mathrm{H}, J=15.7$, 4.3), 2.58-2.65 (m, 1H), $3.54(\mathrm{~s}, 3 \mathrm{H}), 3.66(\mathrm{ddd}, 1 \mathrm{H}, J=11.3,8.3,4.2), 3.82(\mathrm{~s}, 3 \mathrm{H}), 4.05(\mathrm{dt}, 1 \mathrm{H}$, $J=9.9,4.5), 4.46(\mathrm{~d}, 1 \mathrm{H}, J=6.1), 5.48(\mathrm{~d}, 1 \mathrm{H}, J=6.1), 6.15(\mathrm{~s}, 1 \mathrm{H}), 6.51(\mathrm{~s}, 1 \mathrm{H}), 7.13(\mathrm{t}, 1 \mathrm{H}$, $J=7.3), 7.20(\mathrm{t}, 3 \mathrm{H}, J=7.6), 7.25-7.30(\mathrm{~m}, 4 \mathrm{H}), 7.34(\mathrm{~d}, 2 \mathrm{H}, J=7.6) ;{ }^{13} \mathrm{C}-\mathrm{NMR}(150.9 \mathrm{MHz})$ : $147.3,146.5,142.8,141.2,129.5,129.3,128.4,121.9,126.8,126.4,126.1,111.1,109.0,77.5$, 62.6, 57.3, 55.7, 55.5, 28.5. MS m/z: 360 (M+); Anal. Calcd. for $\mathrm{C}_{24} \mathrm{H}_{24} \mathrm{O}_{3}$ : C 79.97, H 6.71. Found: C 79.86, H 6.83 .

6-Methoxy-1-benzhydrylisochroman (5o). ${ }^{1} \mathrm{H}-\mathrm{NMR}(600 \mathrm{MHz}): 2.50$ (dt, 1H, J=15.9, 3.2), 2.62-2.67 (m, 1H), $3.64(\mathrm{ddd}, 1 \mathrm{H}, J=11.0,10.1,3.5), 3.74$ (s, 3H), $4.06(\mathrm{ddd}, 1 \mathrm{H}, J=11.1,5.1$, $3.5), 4.52$ (d, 1H, $J=4.4), 5.48$ (d, 1H, $J=4.4), 6.53$ (d, 1H, $J=2.3), 6.63$ (dd, $1 \mathrm{H}, J=8.6,2.6), 6.85$ (d, 1H, $J=8.6), 7.09$ (t, 1H, $J=7.2), 7.14$ (t, 2H, $J=7.4), 7.18-7.23$ (m, 3H), 7.30 (t, 2H, $J=7.6)$, 7.40 (d, 2H, J=7.7); ${ }^{13} \mathrm{C}-\mathrm{NMR}$ (150.9 MHz): 157.7, 143.1, 140.8, 136.3, 130.0, 129.2, 129.1, 128.2, 127.6, 126.7, 126.3, 126.0, 113.1, 112.0, 78.1, 63.3, 56.9, 55.1, 29.4. MS m/z: $330(\mathrm{M}+)$; Anal. Calcd. for $\mathrm{C}_{23} \mathrm{H}_{22} \mathrm{O}_{2}$ : C 83.60, H 6.71. Found: C 83.72, H 6.82.

\section{Acknowledgements}

We acknowledge financial support from the Fund for Scientific Research of the Plovdiv University.

\section{References}

1. Bogdanov, M. G.; Todorov, I. S.; Manolova, P. G.; Cheshmedzhieva, D. V.; Palamareva M. D. Tetrahedron Lett. 2004, 45, 8383.

2. Horváth, E. J.; Horváth, K.; Hámori, T.; Fekete, M. I. K.; Sólyom, S.; Palkovits, M. Progress in Neurobiology 2000, 60, 309.

3. Gitto, R.; Zappalá, M.; De Sarro, G.; Chimirri, A. Il Farmaco 2002, 57, 129.

4. Chimirri, A.; De Sarro, G.; De Sarro, A.; Gitto, R.; Grasso, S.; Quartarone, S.; Zappalá, M.; Giustri, P.; Libri, V. Constanti, A.; Chapman, A. J. Med. Chem. 1997, 40, 1258. 
5. Nergardh, R.; Oerther, S.; Fredholm, B. B. Pharmacol., Biochem. Behav. 2005, 82, 495.

6. de Vries, P.; Villalón, C. M.; Saxena, P. R. Eur. J. Pharmacol. 1999, 375, 61.

7. Len, C.; Pilard, S.; Lipka, E.; Vaccher, C.; Dubois, M.-P.; Shrinska, Y.; Tran, V. Rabiller, C. Tetrahedron 2005, 61, 10583.

8. de Koning, C. B.; Michael, J. P.; van Otterlo, W. A. L. J. Chem. Soc., Perkin Trans. 12000 , 799.

9. Kashiwaba, N.; Morooka, S.; Kitamura, M.; Ono, M.; Suzuki, H.; Sano, T. J. Nat. Prod. 1996, 59, 803 .

10. Cameron, D. W.; Cromartie, R. I. T.; Kingston, D. G. I.; Todd, R. A. J. Chem. Soc. 1964, 51.

11. Bianchi, D. A.; Blanco, N. E.; Carrillo, N.; Kaufman, T. S. J. Agric. Food Chem. 2004, 52, 1923.

12. Cutler, H. G.; Majetich, G.; Tian X. R.; Spearing, P. J. Agric. Food Chem. 1997, 45, 1422.

13. Liu, J.; Birzin, E. T.; Chan, W. D.; Yang, Y. T.; Pai, L-Y; DaSilva, C.; Hayes, E. C.; Mosley, R. T.; DiNinno, F.; Rohrer, S. P.; Schaeffer, J. M.; Hammond M. L. Bioorg. Med. Chem. Lett. 2005, 15, 715.

14. Tenbrink, R. E.; Bergh, C. L.; Duncan, J. N.; Harris, D. W.; Huff, R. M.; Lahti, R. A.; Lawson, C. F.; Lutzke, B. S.; Martin, I. J.; Rees, S. A.; Schlachter, S. K.; Sih, J. C.; Smith, M. W. J. Med. Chem. 1996, 39, 2435.

15. Fráter, G.; Muller, U.; Kraft, P. Helv. Chim. Acta 1999, 82, 1656.

16. Lorenz, P.; Zeh, M.; Martens-Lobenhoffer, J.; Schmidt, H.; Wolf, G.; Horn, T. F. W. Free Radical Res. 2005, 39, 535.

17. Malmstrom, J.; Christophersen, C.; Frisvad, J. C. Phytochemistry 2000, 54, 301.

18. Togna, G. I.; Togna, A. R.; Franconi, M.; Marra, C.; Guiso, M. J. Nutr. 2003, 133, 2532.

19. Dobson, T. A.; Humber, L. G. J. Heterocyclic Chem. 1975, 12, 591.

20. Yamato, M.; Hashigaki, K.; Ishikawa, S.; Kokubu, N.; Inoue, Y.; Tsuruo, T.; Tashiro, T. J. Med. Chem. 1985, 28, 1026.

21. McCall, J. M.; McCall, R. B.; TenBrink, R. E.; Kamdar, B. V.; Humphrey, S. J.; Sethy, V. H.; Harris, D. W.; Daenzer, C. J. Med. Chem. 1982, 25, 75.

22. Xu, Y.-C.; Lebeau, E.; Attardo, G.; Meyers, P. L.; Gillard, J. W. J. Org. Chem. 1994, 59, 4868.

23. Imamura, N.; Ishikawa, T.; Ohtsuka, T.; Yamamoto, K.; Dekura, M.; Fukami, H.; Nishida, R. Biosci. Biotechnol. Biochem. 2000, 64, 2216.

24. Ogawa, A.; Murakami, C.; Kamisuki, S.; Kuriyama, I.; Yoshida, H.; Sugawara, F.; Mizushina, Y. Bioorg. Med. Chem. Lett. 2004, 14, 3539.

25. Palmquist, U.; Nilsson, A.; Pettersson, T.; Ronlán, A.; Parker, V. D. J. Org. Chem. 1979, 44, 196.

26. Larghi, E. L.; Kaufman, T. S. Synthesis 2006, 0187.

27. Quallich, G. J.; Makowski, T. W.; Sanders, A. F.; Urban, F. J.; Vazquez, E. J. Org. Chem. 1998, 63, 4116.

28. Bhide, B. H.; Shan, K. K. Indian J. Chem., Sect. B 1980, 19, 9. 
29. Mohler, D. L.; Thompson, D. W. Tetrahedron Lett. 1987, 28, 2567.

30. Giles, R. G. F.; Rickards, R. W.; Senanayake, B. S. J. Chem. Soc., Perkin Trans. 1 1997, 3361.

31. Bräse, S. Tetrahedron Lett. 1999, 40, 6757.

32. de Koning, C. B.; Giles, R. G. F.; Green, I. R.; Jahed, N. M. Tetrahedron 2004, 60, 2629.

33. de Koning, C. B.; Green, I. R.; Michael, J. P.; Oliveira, J. R. Tetrahedron 2001, 57, 9623.

34. Anderson, J. R.; Edwards, R. L.; Whalley, A. J. S. J. Chem. Soc., Perkin Trans. 1 1983, 2185.

35. Yamato, M.; Ishikawa, T.; Nagamatsu, T.; Yoshikawa, S.; Koyama, T. Chem. Pharm. Bull. 1980, $28,723$.

36. Donner, C. D.; Gill, M. J. Chem. Soc., Perkin Trans. 1 2002, 938.

37. Fitzgerald, J. J.; Pagano, A. R.; Sakoda, V. M.; Olofson, R. A. J. Org. Chem. 1994, 59, 4117.

38. Moorthy, J. N.; Mal, P.; Singhal, N.; Venkatakrishnan, P.; Malik, R.; Venugopalan, P. J. Org. Chem. 2004, 69, 8459.

39. Endo, K.; Takahashi, H.; Aihara, M. Heterocycles 1996, 42, 589.

40. Bringmann, G.; Breuning, M.; Endress, H.; Vitt, D.; Peters, K.; Peters, E. M. Tetrahedron 1998, 54, 10677.

41. Suzuki, T.; Tanemura, K.; Horaguchi, T.; Kaneko, K. Tetrahedron 2006, 62, 3739.

42. Azzena, U.; Demartis, S.; Fiori, M. G.; Melloni, G.; Pisano, L. Tetrahedron Lett. 1995, 36, 8123.

43. Almena, J.; Foubelo, F.; Yus, M. Tetrahedron 1995, 51, 3351.

44. Guiso, M.; Marra, C.; Cavarischia, C. Tetrahedron Lett. 2001, 42, 6531.

45. Bianchi, D. A.; Rúa, F.; Kaufman, T. S. Tetrahedron Lett. 2004, 45, 411.

46. Sotomayor, N.; Dominguez, E.; Lete, E. J. Org. Chem. 1996, 61, 4062.

47. Antonioletti, R.; Bovicelli, P.; Crescenzi, B.; Luoattelli, P. Tetrahedron Lett. 1998, 39, 6751.

48. Venkov, A. P.; Ivanov, I. I. Synth. Commun. 1994, 24, 1145.

49. Hudlický, M. Reductions in Organic Chemistry, Ellis Horwood Limited, Wiley \& Sons, New York, 1996 , p.156. 